\title{
A comparison of the pharmacokinetics of Aspen Ceftriaxone and Rocephin in community-acquired meningitis
}

\author{
G A Richards, ${ }^{1} \mathrm{MB}$ BCh, FCP (SA), PhD; E Elliott, ${ }^{2} \mathrm{MB}$ ChB, MMed, FCPath (Micro); E J Shaddock, ${ }^{1}$ MB BCh, FCP (SA), Cert Pulm, Cert Crit Care \\ D Mushi, ${ }^{3} \mathrm{MB} \mathrm{ChB}$, MMed; M Mzileni, ${ }^{3} \mathrm{MB} \mathrm{ChB}, \mathrm{MMed}$; R Ray, ${ }^{1} \mathrm{MB}$ BCh, FCP (SA), Cert Pulm; S Rulisa, ${ }^{4} \mathrm{MB}$ ChB, MMed; F Seolwane, ${ }^{3} \mathrm{MB}$ ChB; \\ S L Stacey, ${ }^{1}$ MB BCh, FCP (SA), Cert Inf Dis; A Stolz, ${ }^{5}$ MB ChB, MMed, MSc, PhD; J P Venturas, ${ }^{1}$ MB ChB, FCP (SA), Cert Pulm, Cert Crit Care; \\ H Schoeman, ${ }^{6} \mathrm{DSc}, \mathrm{PrSciNat}$ \\ ${ }^{1}$ Division of Pulmonology and Critical Care, Department of Medicine, University of the Witwatersrand and Charlotte Maxeke \\ Johannesburg Academic Hospital, Johannesburg, South Africa \\ ${ }^{2}$ Department of Microbiology, University of the Free State, Bloemfontein, South Africa \\ ${ }^{3}$ Department of Internal Medicine, School of Medicine, University of Limpopo, MEDUNSA Campus, Pretoria, South Africa \\ ${ }^{4}$ Department of Research, University Teaching Hospital of Kigali, Rwanda \\ Division of Infectious Diseases, University of Pretoria and Department of Internal Medicine, Steve Biko Academic Hospital, Pretoria, South Africa \\ ${ }^{6}$ ClinStat, Pretoria, South Africa
}

Corresponding author: G A Richards (guy.richards@wits.ac.za)

Background. Community-acquired bacterial meningitis (CABM) is a life-threatening condition that is common among immunocompromised individuals. Intravenous ceftriaxone, of which Rocephin (ROC) is the originator brand, is recommended as first-line therapy in South Africa. Despite concerns regarding therapeutic equivalence with generic agents, this is the first study that has been conducted comparing clinical pharmacokinetics (PK) of a generic ceftriaxone formulation with the originator.

Objective. To compare the PK and safety of Aspen Ceftriaxone (AC) and ROC in the treatment of adult CABM.

Methods. A total of 63 eligible patients were randomised 1:1 to receive $2 \mathrm{~g}$ of either medication twice daily for a duration based on the identity of the causative organism and their physician's clinical judgment. The primary endpoint of this study was the comparison of clinical $\mathrm{PK}$, specifically the concentrations of each drug in the cerebrospinal fluid with corresponding paired plasma samples. While this study was underpowered to assess efficacy, safety could be evaluated on the basis of reported adverse events.

Results. The two patient groups were epidemiologically similar. There were no statistically significant differences in PK between either agent, nor any difference with regard to safety.

Conclusion. AC can be considered as equivalent to ROC with regard to PK and safety in patients with CABM.

S Afr Med J 2013;103(12):906-909. DOI:10.7196/SAMJ.7086

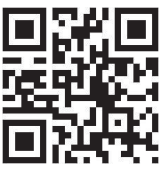

The main causes of community-acquired bacterial meningitis (CABM) in immunocompetent patients are Streptococcus pneumoniae, Neisseria meningitidis, Haemophilus influenzae and, less commonly, Listeria monocytogenes. In immunocompromised patients, specifically those with HIV/AIDS, S. pneumoniae remains the most frequently isolated organism. ${ }^{[1,2]}$

Ceftriaxone is currently recommended as first-line therapy in South Africa (SA) if the aetiology is unknown, because adequate concentrations in the cerebrospinal fluid (CSF) are achieved and it is effective against the organisms described above (with the exception of L. monocytogenes).$^{[3,4]}$ According to the Clinical Laboratory Standards Institute, S. pneumoniae is sensitive to ceftriaxone if the minimum inhibitory concentration (MIC) is $\leq 1 \mu \mathrm{g} / \mathrm{ml}$, it has intermediate resistance if MIC is $2 \mu \mathrm{g} / \mathrm{ml}$ and high-level resistance if the MIC is $\geq 4 \mu \mathrm{g} / \mathrm{ml}$. This would imply that for efficacy the levels in the CSF should exceed $1 \mu \mathrm{g} / \mathrm{ml}$, and preferably $2 \mu \mathrm{g} / \mathrm{ml}$, for at least $50 \%$, but preferably $100 \%$ of the treatment time. ${ }^{[5,6]}$

The original formulation is Rocephin (ROC) (Hoffman-La Roche, Basel Switzerland). One of the many generic preparations is Aspen Ceftriaxone (AC). Registration of generic antibiotics with the SA Medicines Control Council (MCC) requires proof of equivalence with regard to active pharmaceutical ingredient. This internationally accepted standard does not require clinical studies to show efficacy or activity at the target site ${ }^{[4]}$ Consequently, there are very few studies that compare the pharmacokinetics (PK) of original preparations with generics. Those that are available have shown that some generics in some instances may have significantly inferior activity. ${ }^{\left[{ }^{-10]}\right.}$ This, and the prevalent belief in SA that generic antibiotics are inferior, prompted our study.

\section{Objectives}

- Primary: To compare parenteral AC and ROC concentrations administered as $2 \mathrm{~g} 12$-hourly in plasma/CSF of adults with CABM.

- Secondary: To evaluate the safety of AC through adverse event reporting, and clinical/laboratory data from visit 2 to visit 5 .

\section{Methods}

This randomised, double-blind, parallel group study utilised a noninferiority design and was conducted at three centres - two in SA and one in Rwanda - from May 2010 to September 2011. The protocol was approved by the University of the Witwatersrand Human Research Ethics Committee, as well as the MCC (trial ref. no. 20090906). All patients or their representatives gave informed consent.

Male and female patients $\geq 18$ years of age were eligible if they had confirmed CABM. Patients were excluded if: (i) they had received ceftriaxone $<48$ hours before admission; (ii) a second antibiotic (except vancomycin) was anticipated to be required; (iii) there was 
hypersensitivity to $\beta$-lactam antibiotics; (iv) there was any concurrent systemic disease, other than HIV/AIDS; $(v)$ there was severe renal impairment (creatinine clearance $<30 \mathrm{ml} / \mathrm{min}$ ); (vi) the pathogen was resistant to ceftriaxone; (vii) foreign bodies or anatomical defects that predisposed patients to meningitis were present; (viii) the meningitis was mycobacterial, viral, fungal or parasitic; or (ix) the patient was pregnant.

A lumbar puncture was performed to obtain CSF for microscopy, cell count, glucose, protein, bacterial antigens, culture and antibiotic susceptibility; simultaneously, a blood glucose level was obtained. The dose of $2 \mathrm{~g}$ 12-hourly was selected in accordance with standard SA treatment guidelines and eligible patients were randomised 1:1 to receive either medication. The duration of treatment was dependent on the aetiological organism and clinical judgement of the investigator, and was either $7-10$ or $10-14$ days. The use of concomitant vancomycin was left to the investigator's discretion. The study consisted of 5 visits: visit 1 (screening), visit 2 (randomisation), visit 3 (on treatment: days 2; 7 - 10 or 10 - 14), visit 4 (end of treatment: days 10 - 14) and visit 5 (follow-up: days 42 - 49).

Physicians and subjects were blinded as to which treatment was administered. Non-inferiority was concluded if the lower limit of the $95 \%$ confidence interval of the treatment difference in PK profile, and safety showed no statistical difference.

Disease severity was assessed using the Acute Physiology and Chronic Health Evaluation (APACHE) II score.

\section{PK assessment}

This was achieved by comparing concentrations of each drug in the CSF 1 to 2 hours after the fifth dose (visit 3, day 3), with corresponding plasma samples. A liquid chromatography-tandem mass spectrometry (LC-MS/MS) assay was developed and validated for the determination of ceftriaxone concentrations in CSF over the range of 0.125 - $4.00 \mu \mathrm{g} / \mathrm{ml}$, and in human plasma over the range of $0.5-500 \mu \mathrm{g} / \mathrm{ml}$.

Due to the unavailability of human CSF in quantities required for the preparation of calibration standards and quality controls used for the validation of bioanalytical methods, artificial CSF was prepared. Heparinised plasma was utilised for a similar purpose in the plasma method. The assay methods entailed precipitation of proteins to release ceftriaxone into the supernatant. The supernatant was acidified with $0.1 \%$ formic acid, and samples analysed by liquid chromatographic separation using a Phenomenex Onyx C18 $100 \times 3.0$ mm analytical column. An isocratic mobile phase consisting of $0.2 \%$ trifluoroacetic acid and acetonitrile $(50: 50 \mathrm{v} / \mathrm{v})$ was used at a flow rate of $1000 \mu \mathrm{l} / \mathrm{min}$. The retention time for ceftriaxone was $\sim 0.56 \mathrm{~min}$. An Applied Biosystems API 4000 mass spectrometer at unit resolution in the multiple reaction monitoring (MRM) mode was used to monitor transition of the deprotonated precursor ions $m / z 555$ to the product ions $m / z 396$ for ceftriaxone. Electrospray ionisation (ESI) was used for ion production.

Accuracy and precision were assessed over three consecutive, independent runs, with the assay compared with standards and quality control samples prepared for each of the matrices (i.e. CSF and plasma). In the validation, stability of the analyte was assessed at room temperature, during four freeze-thaw cycles and also during longterm storage at $-70^{\circ} \mathrm{C}$.

\section{Safety assessment}

Spontaneously reported adverse events, physical examination and clinical and laboratory data were collected at each visit.

\section{Statistical tests}

Continuous variables were summarised by sample size, mean \pm standard deviation (SD) and minimum and maximum values. Categorical variables were summarised by frequency counts/percentages. Paired and two-sample $t$-tests and Fisher's exact tests were used to test for the significance of changes in values and parameters as appropriate.

Statistical analysis was performed by ClinStat CC, Pretoria, SA. All analyses were carried out using SAS version 9.2. Analysis and interpretation of the data were strictly objective and in accordance with ethical statistical practice.

\section{Results}

A total of 63 patients (57 from SA and 6 from Rwanda) were randomised. Thirty-one received AC and 32 ROC. All were included in the PK and safety populations. Analysis of the population is shown in the consort diagram in Fig. 1. Demographic profiles are shown in Table 1.

\section{Clinical characteristics}

The baseline vital signs of the two groups were similar. Table 2 shows the APACHE II scores within the first 24 hours post admission.

Certain parameters for the APACHE II scores were assumed. The $\mathrm{PaO}_{2}$ was taken to be normal since no patient had comorbid respiratory disease or required mechanical ventilation. For chronic health points, all subjects received a score of 5 .
Mean \pm SD blood glucose values were $5.88 \pm 2.21 \mathrm{mmol} / \mathrm{l}$ and $6.05 \pm 1.63 \mathrm{mmol} / \mathrm{l}$ and mean \pm SD CSF levels were 2.01 $\pm 1.83 \mathrm{mmol} / \mathrm{l}$ and $2.81 \pm 3.43 \mathrm{mmol} / \mathrm{l}$ at screening for $\mathrm{AC}$ and $\mathrm{ROC}$, respectively. Blood cultures were performed on 15 patients in each group; 6 (40\%) of the AC patients were positive v. $4(26.7 \%)$ in the ROC group.

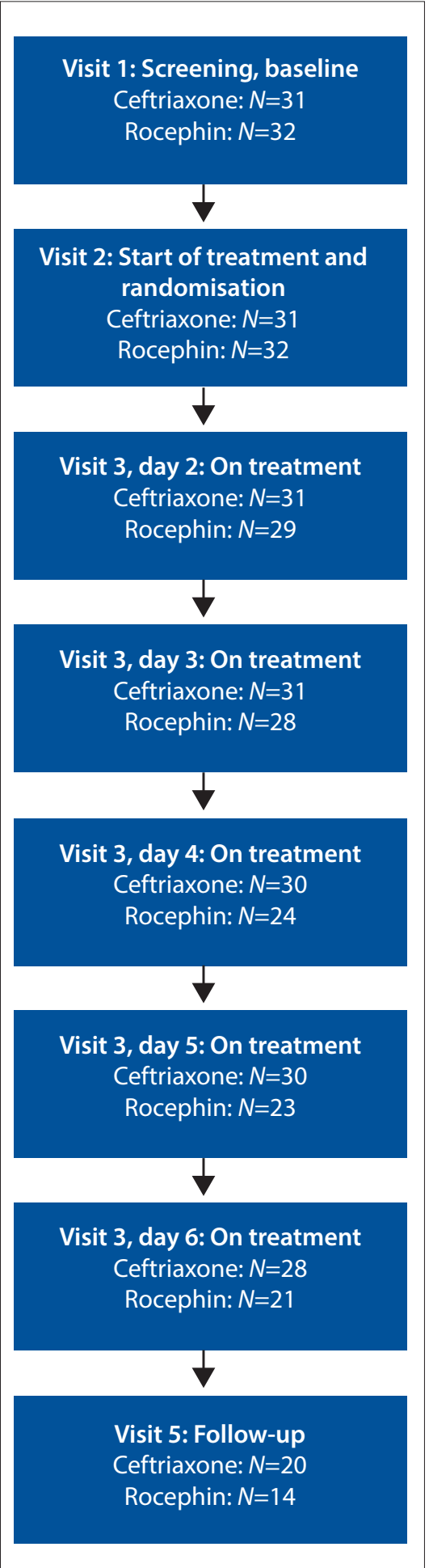

Fig. 1. Consort flow diagram. 
Laboratory confirmation of CABM remains a challenge due to the fastidious nature of many of the organisms; as a result negative cultures are common. In 34 patients there was neither bacterial growth nor evidence of bacterial presence on the final analysis. In 6 patients there was an indication of a bacterial aetiology of meningitis, with a positive Gram stain or neutrophil predominance, but no confirmation on culture. Cultures were positive in 12 patients, of which 9 were S. pneumonia and $3 N$. meningitides. One patient grew an Enterobacter cloacae (which is uncommon, but may reflect the immune-suppressed population in this study). Dual infection with Cryptococcus and Mycobacterium tuberculosis (MTB) occurred in 1 patient each. Cryptococcus (in 3 patients) and MTB (in 1 patient) were cultured as a single organism causing meningitis.

Table 1. Demographic characteristics of subjects

\begin{tabular}{lll}
\hline & $\begin{array}{l}\text { Aspen Ceftriaxone } \\
(N=31)\end{array}$ & $\begin{array}{l}\text { Rocephin } \\
(N=32)\end{array}$ \\
\hline Age (years), mean \pm SD & $36.8 \pm 34$ & $38.6 \pm 36$ \\
Height $(\mathrm{cm})$, mean $\pm \mathrm{SD}$ & $164.9 \pm 11.7$ & $163.3 \pm 11.5$ \\
Weight $(\mathrm{kg})$, mean $\pm \mathrm{SD}$ & $60 \pm 13.6$ & $58.8 \pm 12.3$ \\
Gender (female), $n$ & 15 & 18 \\
$\quad$ Child-bearing age & 14 & 13 \\
Males, $n$ & 16 & 14 \\
$\mathrm{SD}=$ standard deviation & &
\end{tabular}

Table 2. APACHE II scores

\begin{tabular}{lll} 
& Aspen Ceftriaxone & Rocephin $^{*}$ \\
\hline Total, $N$ & 31 & 32 \\
Mean \pm SD & $13.2 \pm 3.6$ & $13.3 \pm 5.4$ \\
Median & 13 & 12.5 \\
Min/max & $8 / 21$ & $7 / 27$ \\
APACHE $=$ Acute Physiology and Chronic Health Evaluation; SD = standard deviation. \\
${ }^{*} p$-value Aspen Ceftriaxone v. Rocephin was not significant.
\end{tabular}

Table 3. Comparison of plasma/CSF levels of Aspen Ceftriaxone and Rocephin in patients

\begin{tabular}{lll}
\hline & \multicolumn{2}{c}{ Patients, $\boldsymbol{n}$ (\%) } \\
\cline { 2 - 3 } Sample/quantity & Aspen Ceftriaxone & Rocephin \\
\hline Plasma $(\mu \mathrm{g} / \mathrm{ml})$ & $22(100)$ & $17(100)$ \\
$>200$ & $3(13.64)$ & $5(29.41)$ \\
$>100-200$ & $9(40.91)$ & $6(35.3)$ \\
$\leq 100$ & $8(36.36)$ & $2(11.76)$ \\
BQL & $2(9.09)$ & $4(23.53)$ \\
CSF $(\mu \mathrm{g} / \mathrm{ml})$ & $24(100)$ & $18(100)$ \\
$>4$ & $11(45.83)$ & $9(50)$ \\
$>2-4$ & $9(37.5)$ & $3(16.67)$ \\
$>1-2$ & $1(4.17)$ & $0(0)$ \\
$\leq 1$ & $0(0)$ & $2(11.11)$ \\
BQL & $3(12.5)$ & $4(22.22)$ \\
CSF $=$ cerebrospinal fluid; BQL $=$ below quantifiable level. &
\end{tabular}

\section{Validation of the assay method}

Validation in artificial CSF was robust, with an accuracy of $-0.3-2.5 \%$ over the range of $0.125-4.00 \mu \mathrm{g} / \mathrm{ml}$, and a precision of $4.4-13.5 \%$ (based on performance of the quality control samples). Similarly, the accuracy was $2.7-7.0 \%$ over $0.5-500 \mu \mathrm{g} / \mathrm{ml}$, and precision $-5.2-2.0 \%$ in plasma.

Ceftriaxone, reported to be stable for up to 52 and 57 days when stored at $-70^{\circ} \mathrm{C}$, was stable in CSF and plasma for 4 and 15 hours, respectively, at room temperature and when subjected to four freeze-thaw cycles.

Endogenous components of plasma had an insignificant effect on reproducibility of the method when human plasma originating from 10 different sources was analysed; the assay was unaffected by haemolysed blood (1\%) in plasma.

The assay met the currently accepted validation standards for analytical methods with regard to bioavailability, bioequivalence and PK studies as outlined by the United States Food and Drug Administration (FDA $)^{[11]}$ and the report from the conference on Quantitative Bioanalytical Methods, Validation and Implementation. ${ }^{[12]}$ As the assay method for the CSF was validated on artificial CSF, some assessments were omitted that would otherwise have been performed had human CSF been available in adequate quantities.

\section{Analytical results}

CSF and plasma levels of both drugs, compared in Table 3, did not differ significantly ( $p=0.182$ for plasma and $p=0.179$ for CSF, Fisher's exact test). As shown in Tables 4 and 5, the Wilcoxon rank test was used to compare medians because the numbers were small and comparison of means by the $t$-test was unreliable. The medians varied significantly in plasma $(p=0.028)$ but not in CSF $(p=0.881)$. The statistical comparisons did not show significant differences.

\section{Safety evaluation/assessment}

Of the 63 subjects, 16 in the ROC and 9 in the AC group experienced serious adverse events. All 16 patients in the ROC group died. Death was not believed to have a causal relationship to the study drug: 9 deaths were due to concurrent illness, 4 to cerebral lesions complicating meningitis and in 3 , cause of death was unknown.

Table 4. Statistics for plasma levels of Aspen Ceftriaxone and Rocephin (excluding BQLs)

\begin{tabular}{lll}
\hline & Aspen Ceftriaxone & Rocephin \\
\hline Total, $N$ & 20 & 13 \\
Mean \pm SD & $122.46 \pm 83.57$ & $176.12 \pm 61.94$ \\
Median & 110 & 184 \\
IQR & $56.35-156$ & $174-223$ \\
Min/max & $22.6 / 317$ & $44.5 / 234$
\end{tabular}

$\mathrm{BQL} s=$ below quantifiable levels; $\mathrm{SD}=$ standard deviation; $\mathrm{IQR}=$ interquartile range.

Table 5. Statistics for CSF levels of Aspen Ceftriaxone and Rocephin (excluding BQLs)

\begin{tabular}{lll}
\hline & Aspen Ceftriaxone & Rocephin \\
\hline Total, $N$ & 21 & 14 \\
Mean \pm SD & $3.50 \pm 0.78$ & $3.26 \pm 1.2$ \\
Median & 4 & 4 \\
IQR & $3.23-4$ & $2.75-4$ \\
Min/max & $1.47 / 4$ & $0.57 / 4$
\end{tabular}

$\mathrm{CSF}=$ cerebrospinal fluid; $\mathrm{BQLs}=$ below quantifiable levels; $\mathrm{SD}=$ standard deviation $\mathrm{IQR}=$ interquartile range. 
In the AC group, there were 7 deaths. Similarly, no death was believed to have a causal relationship to the drug. In 6 patients, death was due to concurrent illness and in 1 due to cerebral lesions complicating meningitis; in 2 there was a probable causal relationship to concomitant medication. One patient suffered a life-threatening event (worsening of meningitis); 1 had a prolonged hospital stay. Only 1 patient in the AC group received vancomycin.

\section{Outcomes}

Of the 31 patients who received AC, 29 completed treatment; 21 recovered fully and were discharged, 7 died and 1 was referred for surgery, remaining in hospital for a craniotomy for evacuation of a haematoma before visit 5 (follow-up).

In the ROC group, 24 patients completed the treatment; 16 recovered fully and were discharged, and 8 died.

In the AC group, 22 patients completed the study; of the 9 that did not, 7 died in hospital, 1 died after discharge and one patient was lost to follow-up. In the ROC group, 15 patients completed the study; 16 did not due to adverse events and 1 was lost to follow-up.

\section{Discussion}

This study evaluated the PK profiles of ROC and AC. Although there was a significant difference in survival between the two groups, numbers were insufficient to eliminate confounding factors such as HIV positivity, degree of immunosuppression, use of antiretrovirals, co-existent disease and delay before presentation. The primary endpoint was, however, achieved in that CSF/plasma levels of the drugs were equivalent and $\mathrm{AC}$ was found to be non-inferior to ROC. Additionally, AC had a similar safety profile to ROC. Although there were more fatalities in the cohort receiving the latter, in none was there a causal relationship to the drug.

The ability of an antimicrobial agent to eradicate pathogens is often predicted by its pharmacodynamic and PK properties. In meningitis the ability of the agent to penetrate the blood-brain barrier and achieve rapid and sustained bactericidal concentrations is the best predictor of bacterial killing. Penetration of the blood-brain barrier is dependent on protein binding, lipophilicity, molecular weight, efflux transporters and the degree of inflammation of the meninges. ${ }^{[1,1,14]}$ Ceftriaxone has adequate penetration and is not affected by efflux transporter systems, making it suitable for central nervous system infections, including meningitis. ${ }^{[15]}$

A recent study in children showed that a $\beta$-lactam infusion along with paracetamol lowered mortality in childhood bacterial meningitis in the first 3 days, but overall mortality was unchanged. ${ }^{[16]}$ This improvement was thought to be related to the prolonged infusion of the antibiotic, rather than the adjuvant paracetamol, because the antibiotics exhibit time-dependent killing and infusion increases the time above the MIC. ${ }^{[17]}$ All of our patients had confirmed meningitis, with evidence of inflammation, but there was a poor correlation between plasma and CSF levels as measured after the fifth dose. In the AC group, 21/24 and $12 / 18$ in the ROC group had CSF levels $>1 \mu \mathrm{g} / \mathrm{ml}$ (Table 3 ). Of particular concern, however, was that significant numbers in both groups had CSF levels below the quantifiable range: $3(12.5 \%)$ and $4(22.2 \%)$ in the AC and ROC groups, respectively. This was despite documented administration of the drug. An additional 2 patients in the ROC group had CSF levels $<1 \mu \mathrm{g} / \mathrm{ml}$, which was likely also to be subtherapeutic. Thus, there were 9 patients with potentially subtherapeutic CSF levels.

These low CSF levels may have been due to errors in the estimation method (despite being performed by means of a validated technique), or due to undocumented administration or transport errors. These are all unlikely, as doses were observed and ampoules collected after each treatment day. It is also possible that augmented renal clearance may have contributed to low levels. This phenomenon has been well described in septic patients and results from an increase in glomerular filtration due to factors such as the increased cardiac output that occurs in early sepsis and the use of catecholamine inotropes. ${ }^{[18,19]}$ Another factor that may have contributed to lower levels is serum albumin, ${ }^{[20,21]}$ which, when low, increases the free fraction and in turn increases the volume of distribution and excretion of highly proteinbound drugs. We were not, however, able to relate the low drug levels to albumin in these patients. We did not measure glomerular filtration rate (GFR) and the calculated creatinine clearance, as a measure of GFR, was inaccurate in this setting.

Approximately $20-60 \%$ of pneumococci in SA have an MIC of $2 \mu \mathrm{g} / \mathrm{ml}$ for penicillin, but there is little resistance to third-generation cephalosporins in either the private or public sectors, with most MICs at $\leq 0.5 \mu \mathrm{g} / \mathrm{ml} .^{[4]}$ This suggests that therapeutic drug monitoring would be the best way to manage these patients, that dosing schedules should be altered or that other agents, such as ceftaroline, should be considered.

The above notwithstanding, the recommended therapy for meningitis is ceftriaxone. Both AC and ROC performed equivalently in this study.

Acknowledgements. Financial assistance for the purchase of trial materials and payment of experimental costs was received, and ROC and AC were provided and paid for, by Aspen Pharmacare Ltd, SA.

Conflict of interest. GAR has delivered academic lectures on behalf of both Aspen and Roche and has served on advisory boards for both companies.

\section{References}

1. Van de Beek D, de Gans J, Spanjaard L, et al. Clinical features and prognostic factors in adults with bacterial meningitis. N Engl J Med 2004;351:1849-1859. [http://dx.doi.org/10.1056/NEJMoa040845] 2. Kongsiriwattanakul S, Suankratay C. Central nervous system infections in HIV-infected patients hospitalized at King Chulalongkorn Memorial Hospital. J Med Assoc Thai 2011;94:551-558.

3. Lepur D, Barsić B. Community-acquired bacterial meningitis in adults: Antibiotic timing in disease course and outcome. Infection 2007;35(4):225-231. [http://dx.doi.org/10.1007/s15010-007-6202-0] SASCM Laboratory Surveillance: Private Sector, July-December 2011. http://www.mm3admin.coza/ documents/docmanager/47f68655-acdf-4462-8366-64afd5633d81/00026116.pdf (accessed 13 June 2012). 5. Li C, Du X, Kuti JL, Nicolau DP. Clinical pharmacodynamics of meropenem in patients with lower respiratory tract infections. Antimicrob Agents Chemother 2007;51(5):1725-1730.

6. McKinnon PS, Paladino JA, Schentag JJ. Evaluation of area under the inhibitory curve (AUIC) and . McKinnon PS, Paladino JA, Schentag JJ. Evaluation of area under the inhibitory curve (AUIC) and
time above the minimum inhibitory concentration (T $>$ MIC) as predictors of outcome for cefepime and ceftazidime in serious bacterial infections. Int J Antimicrob Agents 2008;31(4):345-351. [http:// and ceftazidime in serious bacterial infections. Int J Antimicrob Agents 2008;31(4):345-351. [http://
dx.doi.org/10.1016/j.jiantimicag.2007.12.009]

Schito GC, Keenan MH. Predicting the clinical efficacy of generic formulations of ceftriaxone. J Chemother 2005;17(Suppl 2):33-40.

8. Moet GJ, Watters AA, Sadera HS, Jones RN. Expanded studies of piperacillin/tazobactam formulations: Variations among branded product lots and assessment of 46 generic lots. Diagn Microbiol Infect Dis 2009;65(3):319-322. [http://dx.doi.org/10.1016/j.diagmicrobio.2009.06.012]

9. Mastoraki E, Michalopoulos A, Kriaras J, et al. Incidence of postoperative infections in patients undergoing coronary artery bypass grafting surgery receiving antimicrobial prophylaxis with original and generic cefuroxime. J Infect 2008;56(1):35-39. [http://dx.doi.org/10.1016/j.jinf.2007.09.011]

10. Vesga $\mathrm{O}$, Agudelo M, Salazar BE, Rodriguez CA, Zuluaga AF. Generic vancomycin products fail in vivo despite being pharmaceutical equivalents of the innovator. Antimicrob Agents Chemother 2010;54(8): 3271-3279. [http://dx.doi.org/10.1128/AAC.01044-09]

11. FDA Drug Information Branch (HFD-210), Center for Drug Evaluation and Research (CDER), Center for

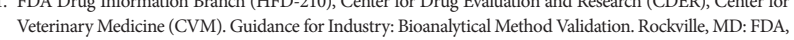
Veterinary Medicine (CVM). Guidance for Industry: Bioanalytical Method Validation. Rockville, MD: FDA,
2001. http://www.fda.gov/downloads/Drugs/../Guidances/ucm070107.pdf (accessed 9 November 2012).

2001. http://www.fda.gov/downloads/Drugs/.../Guidances/ucm070107.pdf (accessed 9 November 2012).
12. Viswanathan CT, Bansal S, Booth B, et al. Quantitative bioanalytical methods validation and implementation: Best practices for chromatographic and ligand binding assays. Pharm Res
iswanathan CT, Bansal S, Booth B, et al. Quantitative bioanalytical methods validation and 2007;24(10):1962-1973. [http://dx.doi.org/10.4172/2155-9872.S4-001]

13. Kim KS. Acute bacterial meningitis in infants and children. Lancet Infect Dis 2010;10(1):32-42. [http:// dx.doi.org/10.1016/S1473-3099(09)70306-8]

14. Nau R, Sorgel F, Eiffert H. Penetration of drugs through the blood-cerebrospinal fluid/blood-brain barrier for the treatment of central nervous system infections. Clin Microbiol Rev 2010;23(4):858-883. [http://dx.doi.org/10.1128/CMR.00007-10]

15. Spector R. Nature and consequences of mammalian brain and CSF efflux transporters: Four decades of progress. J Neurochem 2010;112(1):13-23. [http://dx.doi.org/10.1111/j.1471-4159.2009.06451.x]

16. Pelkonen T, Roine I, Cruzeiro ML, Pitkäranta A, Kataja M, Peltola H. Slow initial $\beta$-lactam infusion and oral paracetamol to treat childhood bacterial meningitis: A randomised, controlled trial. Lancet and oral paracetamol to treat childhood bacterial meningitis: A randomised, contro

17. MacGowan A. Revisiting beta-lactams - PK/PD improves dosing of old antibiotics. Curr Opin MacGowan A. Revisiting beta-lactams - PK/PD improves dosing of old a
Pharmacol 2011;11(5):470-476. [http://dx.doi.org/10.1016/j.coph.2011.07.006]

18. Baptista JP, Udy AA, Sousa E, et al. A comparison of estimates of glomerular filtration in critically ill paptista JP, Udy AA, Sousa E, et al. A comparison of estimates of glomerular filtration in critically ill
patients with augmented renal clearance. Crit Care 2011;15:R139 [http:///dx.doi.org/10.1186/cc10262] patients with augmented renal clearance. Crit Care $2011 ; 15: R 139$ [http://dx.doi.org/10.1186/cc10262]
9. Udy AA, Roberts JA, Boots R, et al. Augmented renal clearance implications for antibacterial dosing in the critically ill. Clin Pharmocokinet 2010;49(1):1-16. [http://dx.doi.org/10.2165/11318140-000000000-00000] 20. Liu P, Derendorf H. Antimicrobial tissue concentrations. Infect Dis Clin North Am 2003;17(3):599613. [http://dx.doi.org/10.1016/S0891-5520(03)00060-6]

21. Roberts JA, Hope WW, Lipman J. Therapeutic drug monitoring of $\beta$-lactams for critically ill patients: Unwarranted or essential? Int J Antimicrob Agents 2010;35(5):419-420. [http://dx.doi.org/10.1016/j. ijantimicag. 2010.06.008]

Accepted 25 July 2013 